
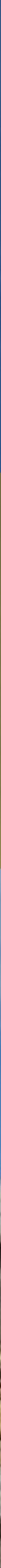


\section{Panta Rei \\ Revista Digital de Ciencia \\ y Didáctica de la Historia}

\section{6}

Revista anual

Fecha de inicio: 1995

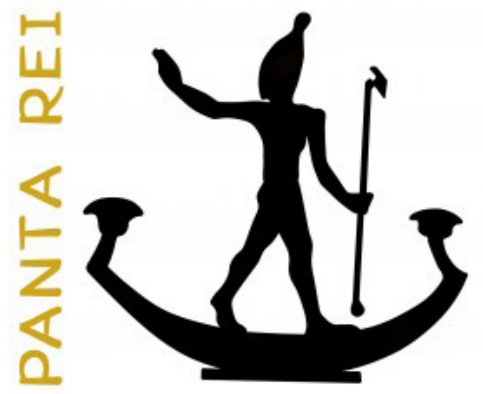

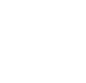




\section{CONSEJO DE REDACCIÓN}

\section{Coordinador editorial}

Egea Vivancos, Alejandro

[Didáctica de las Ciencias Sociales, UMU]

\section{Editores}

Botí Hernández, Juan Jesús

[CEPOAT, UMU]

Meseguer Gil, Antonio José

[CEPOAT, UNED]

Sáez Giménez, David Omar

[CEPOAT, UMU]

Sánchez Mondéjar, Celso Miguel

[CEPOAT, UMU]

\section{Secretaria}

Arias Ferrer, Laura

[Didáctica de las Ciencias Sociales, UMU]

\section{Responsable informático}

Martínez García, José Javier

[CEPOAT, UMU]

\section{Traducción y corrección lingüística}

Martínez Martínez, Cristina

[Sociedad Española de Lenguas Modernas]

Albaladejo Albaladejo, Sara

[ISEN, UMU]

\section{CONSEJO ASESOR}

Albero Muñoz, M. ${ }^{a}$ del Mar

[H. ${ }^{a}$ del Arte, UMU]

Chapman, Arthur

[History Education, UCL, Reino Unido]

Cobacho López, Ángel

[Derecho, UMU]

Egea Bruno, Pedro M. ${ }^{\text {a }}$

[Historia Contemporánea, UMU]

García Atienzar, Gabriel

[Prehistoria, UA]

González Monfort, Neus

[Didáctica de las Ciencias Sociales, UAB]

Haber Uriarte, María

[Prehistoria, UMU]

Hutson, Scott R.

[Anthropology, UK, EEUU]

Irigoyen López, Antonio

[Historia Moderna, UMU]

Mahony, Simon

[Digital Humanities, UCL, Reino Unido]

Marsilla de Pascual, Francisco Reyes

[Técnicas historiográficas, UMU]

Miralles Maldonado, José Carlos

[Filología Clásica, UMU]

Molina Gómez, José Antonio

[Historia Antigua, UMU]

Noguera Celdrán, José Miguel

[Arqueología, UMU]

Pérez Molina, Miguel Emilio

[Filología Clásica, UMU]

Prados Martínez, Fernando

[Arqueología, UA]

Sánchez Ibáñez, Raquel

[Didáctica de las Ciencias Sociales, UMU]

Sancho Gómez, Miguel Pablo

[Educación, UCAM]

Vilar García, María José

[Historia Contemporánea, UMU]

Zamora López, José Ángel

[Próximo Oriente Antiguo, CCHS-CSIC] 

Artículos

Los orígenes de la tecnología a debate: una revisión de las primeras industrias líticas.

Arturo Cueva Temprana.

De arqueología menorquina: Maria Lluïsa Serra Belabre y los círculos talayóticos de Sant Vicenç d' Alcaidús (Alaior, Menorca).

Octavio Torres Gomariz.

Nuevas cuestiones sobre el anfiteatro de Zaragoza.

José David Mendoza Álvarez.

Castidad o castigo. El estupro de las Vestales como símbolo de desorden social en Roma.

Juan Antonio Montalbán Carmona.

La Historia antigua en la Biblioteca de Focio.

Juan Luis Posadas Sánchez.

Usos sociales de la historia. La estrategia de Olga Cossettini, Rosario, 1935-1943.

Paula Caldo, Micaela Pellegrini Malpiedi y Agustina Mosso

Contribuciones a la didáctica de la Historia a través del método de análisis del objeto: como ejemplo... una "vasulla".

Nayra Llonch Molina y Verónica Parisi Moreno.

How are digital methods changing research in the study of the classical world? An EpiDoc case study. Katherine Steiner y Simon Mahony.

\section{Reseñas}

I Congreso Internacional "Creando ciudadanos, construyendo identidades. El uso del patrimonio material e inmaterial en la enseñanza de la historia."

José Díaz Serrano, Ainoa Escribano Miralles, Ana Isabel Ponce Gea y David Verdú González 151

Beckert, S. (2014). Empire of cotton: A global history. New York: Alfred A Knopf. 640 págs.

Ricky D. Mullins Jr..

Coumert, M. y Dumézil, B. (2013): Los reinos bárbaros en Occidente (traducción de Peinado Santaella, R. G.: Les royaumes barbares en Occident, Presses Universitaires de France, 2010). Editorial Universidad de Granada. Granada. 156 págs.

José Ángel Castillo Lozano.

La prehistoria en Las tres edades de Buster Keaton.

Alberto Lombo Montañés y Esther Rodríguez Ortiz.

Normas de publicación/Publishing rules 



\title{
Los orígenes de la tecnología a debate: una revisión de las primeras industrias líticas
}

\author{
The origins of technology under debate: a review of the first lithic \\ industries
}

Recibido: 31/05/2016

Aceptado: 28/08/2016

Para citar este artículo: Cueva Temprana, A. (2016). Los orígenes de la tecnología a debate: una revisión de las primeras industrias líticas. Panta Rei. Revista Digital de Ciencia y Didáctica de la Historia, 9-18.

ISSNe: 2386-8864

DOI: $10.6018 /$ pantarei/2016/1

\begin{abstract}
Resumen
Durante muchos años, la comunidad científica ha considerado la producción de artefactos líticos como privativa del género Homo. Sin embargo, recientes hallazgos realizados en yacimientos africanos han aportado unos resultados que parecen poner en duda esta cuestión, retrasando la cronología de aparición de las primeras industrias líticas hasta más allá de los $2.6 \mathrm{Ma}$. planteados por el yacimiento de Gona. A partir de una revisión de estos yacimientos, el presente trabajo pretende realizar una reflexión sobre la posible autoría de estas primeras tecnologías, enmarcadas en un contexto paleoantropológico de creciente complejidad, y quizás atribuibles a miembros de otros géneros.
\end{abstract}

\section{Palabras clave}

Antropología, Arqueología, Desarrollo Cognitivo, Prehistoria, Avance Tecnológico.

\begin{abstract}
For many years, the scientific community has considered the production of lithic artifacts as a unique characteristic of the genus Homo. However, recent discoveries made in African sites have provided results that seem to put this issue in doubt, delaying the appearance time of the first stone industries to beyond the 2.6 Myr. posed by the deposits of Kada Gona. This paper aims to make a review on these sites, trying to consider the possible authorship of that early technologies, which are framed in a high complexity paleoanthropological context, and perhaps are attributable to members of other genres.
\end{abstract}

\section{Keywords}

Anthropology, Archaeology, Cognitive Development, Prehistory, Technological Development.

1 Para contactar con el autor: Arturo Cueva Temprana. Universitat Rovira i Virgili. arturocuevatemprana@ gmail.com 


\section{Introducción}

La génesis de la investigación sobre los orígenes de la tecnología discurre de forma paralela a la de la propia arqueología prehistórica. Ya desde principios del siglo XX, la lucha por el descubrimiento de las cronologías más antiguas ha sido un tema recurrente y cargado de controversia (de la Torre, 2011). Considerado como uno de los principales criterios definitorios de Homo, la fabricación de herramientas se entiende, pues, íntimamente ligada a nuestro género.

Fue, en efecto, la asignación de restos fósiles humanos al taxón Homo habilis (Leakey, Tobias, Napier, 1964) lo que estableció dicho paradigma, claramente influenciado por el concepto de cultura, privativa del ser humano, que era manejado en la época (White, 1959). Aceptando esta premisa, únicamente los miembros del género Homo tendrían la capacidad de generar industria lítica. Como consecuencia, África, pese a discrepancias iniciales, cobró protagonismo como cuna de la humanidad y se convirtió en el continente que proporcionó la tecnología más antigua asociada a una autoría definida. El devenir de la investigación ha generado, con los años, que el mapa paleoantropológico de nuestro género haya tornado en una remarcable complejidad. En este trabajo, por tanto, se utilizará el término Homo habilis en sentido genérico para referirse a la especie más antigua del género Homo, teniendo en cuenta, sin embargo, la problemática de su denominación y la relación de esta especie con Homo rudolfensis y la línea de los australopitecinos (Gómez Castanedo e Yravedra, 2009; Schwartz y Tattersall, 2015).

Además, la sistematización realizada por los Leakey a raíz de los trabajos en Olduvai sirvió para que la concepción del tecnocomplejo Olduvayense como la más arcaica manifestación cultural cristalizase en la comunidad científica (de la Torre, 2011). El hallazgo de los conjuntos de Kada Gona y Ounda Gona (Semaw, 2000; Semaw, Renne, Harris, Feibel, Bernor, Fesseha y Mowbray, 1997; Semaw et al. 2003) en las décadas de 1990 y 2000, aunque ya conocidos desde los años 70 , supuso quizás el clímax de este planteamiento: la cronología de los 2,5-2,6 Ma. que presenta el yacimiento encaja a la perfección con la idea de una producción industrial ligada a los estadios más tempranos de nuestro género, al situarse Homo habilis en un marco temporal ligeramente posterior. Por otro lado, el reciente hallazgo en Ledi-Geraru (Afar, Etiopía) retrasa la evidencia más antigua de presencia del género Homo hasta los 2.8 Ma. (Villmoare et al., 2015). En la misma línea se enmarcan los hallazgos, entre otros, Lokalalei 2C (West Turkana, Kenya) (Delagnes y Roche, 2005) con industrias que presentan una cronología de 2.3-2.4 Ma. o Kanjera South (Kenya) (Plummer, Bishop, Ditchfield y Hicks, 1999) con industrias de $2.2 \mathrm{Ma}$.

Sin embargo, la arqueología no es una disciplina inmutable y un hallazgo como el descubrimiento de Australopithecus garhi (Asfaw, White, Lovejoy, Latimer, Simpson y Suwa, 1999), en la localidad de Bouri (Middle Awash, Etiopía) y con una cronología de $2.5 \mathrm{Ma}$., sirvió para que una evidencia, en este caso indirecta, a partir de la asociación territorial de los fósiles con fauna procesada (de Heinzelin et al. 1999), sembrase la duda sobre la autoría de estas primeras industrias. Las recientes publicaciones sobre marcas de corte encontradas en restos de fauna recuperados en Dikika (Lower Awash, Etiopía) y el conjunto de herramientas líticas de Lomekwi (West Turkana, Kenya), ambos descubrimientos con cronologías que podrían rondar los 3.3 Ma., han reabierto el debate sobre la antigüedad y la autoría de la primera tecnología, permitiendo recrudecer una discusión a la que se añaden argumentos que retrasan 800.000 años el origen hasta la fecha aceptado y planteando un nuevo horizonte de posibilidades.

La idea de una tecnología anterior a los artefactos de piedra que conocemos no es nueva, y ya ha sido tratada en la bibliografía (Panger, Brooks, Richmond y Wood, 2002). Es más, ya desde el siglo XIX autores como Marcellin Boule teorizaban sobre la existencia de un hipotético estadio previo a la fabricación de herramientas, un Eolítico (de la Torre, 2011), donde el aprovechamiento de formas y filos naturales se habría consolidado como fase anterior a la producción de artefactos líticos tal y como la concebimos en la actualidad. En la misma línea argumental se posicionaba Raymond Dart cuando exponía su tesis sobre el osteodontoqueratismo (Dart, 1957; Dart y Wolberg, 1971). Sirvan estas pinceladas para comprender que esta es una noción largo tiempo arraigada en 
la comunidad arqueológica.

Además, esta idea ha sido contemplada por los estudios primatológicos y etoarqueológicos prácticamente desde la misma aparición de la disciplina. Como ejemplo, en 1978 Jordi Sabater Pi publicaba El chimpancé y los orígenes de la cultura, dotando a esta argumentación de impacto y difusión internacional. En ese sentido, a lo largo de las últimas décadas se han desarrollado trabajos que abogan por el estudio de chimpancés (Pan troglodytes) y bonobos (Pan paniscus) desde el punto de vista de su cultura material (Carvalho, Cunha, Sousa y Matsuzawa, 2008; Carvalho, Biro, McGrew y Matsuzawa, 2009; Haslam et al. 2009; Inoue-Nakamura y Matsuzawa 1997; McGrew, 1992; Mercader, Panger y Boesch, 2002), particularmente focalizados en el estudio de actividades manuales complejas tales como el nut-cracking, conscientes de que su proximidad filogenética con la subtribu Hominina se encuentra repleta de riqueza informativa sobre los orígenes de la tecnología.

El presente trabajo pretende, por tanto, indagar en estas cuestiones mediante una revisión de los yacimientos que se encuentran en primera línea de discusión y plantear las posibles opciones sobre la autoría de las industrias más antiguas, a partir de inferencias debidamente apoyadas en su correspondiente vaciado bibliográfico.

\section{Yacimientos}

\subsection{Gona}

El entorno de Gona (Afar, Etiopía) ha proporcionado yacimientos clave en lo referente a la investigación sobre los orígenes de la tecnología. Las excavaciones realizadas en East Gona entre los años 1992 y 1994 dieron como resultado el hallazgo de industrias datadas en 2.5-2.6 Ma. en EG-10 y EG-12, con un conjunto compuesto por más de 3000 artefactos líticos recuperados tanto en superficie como en excavación (Semaw, 2000; Semaw et al., 1997, 2003).

Enmarcados en el mismo proyecto se desempeñaron los trabajos en Ounda Gona, los cuales derivaron en la recuperación de más de doscientas piezas líticas in situ y otras quinientas en superficie, variando la naturaleza del yacimiento, en este caso, al encontrarse restos de fauna entre las mismas. Las dataciones referidas a estos sitios, OG-6 y OG-7, corroboran las obtenidas para EG-10 y EG-12, con cronologías de $2.6 \mathrm{Ma}$. obtenidas mediante magnetoestratigrafía y ${ }^{40} \mathrm{Ar} /{ }^{39} \mathrm{Ar}$, mismos procedimientos empleados que en el caso anterior (Semaw et al., 2003).

Los materiales recuperados en ambos sitios se corresponden con un modo técnico 1 que en este caso queda bien ejemplificado a partir de la presencia de núcleos explotados unifacial y bifacialmente, lascas simples (tanto enteras como fracturadas) y debrises. Las materias primas predominantemente explotadas son el chert y los conglomerados volcánicos de la zona, esencialmente traquitas, riolitas y latitas (Semaw et al. 2003). Su aprovisionamiento, sin embargo, no se encuentra condicionado por la cercanía de su disponibilidad, sino que se ha comprobado un proceso de selección de la materia en busca de aquella de mejor calidad, de cara a abordar el proceso de talla (Stout, Quade, Semaw, Rogers y Levin, 2005).

El Modo 1 (Clark, 1977; Díez Martín, 2003) se caracteriza por estar orientado a la producción de lascas a partir de cadenas operativas cortas y sistemas de talla de poca complejidad, manifestando además una escasa presencia de objetos retocados. A pesar de mantener estas características arcaicas, los conjuntos de Gona presentan una sofisticación fuera de lo común, si se tiene en cuenta la cronología a la cual se encuentran adscritas. Ello ha permitido inferir la existencia de una tecnología previa, a partir de la cual se haya evolucionado hasta adquirir el nivel de control técnico que presentan los materiales recuperados en los yacimientos de Gona. En sitios como Lokalalei 2C se han obtenido conclusiones similares (Delagnes y Roche, 2005) también a partir de la identificación de una complejidad inusitada para la cronología de 2.3-2-4 Ma. del yacimiento. 

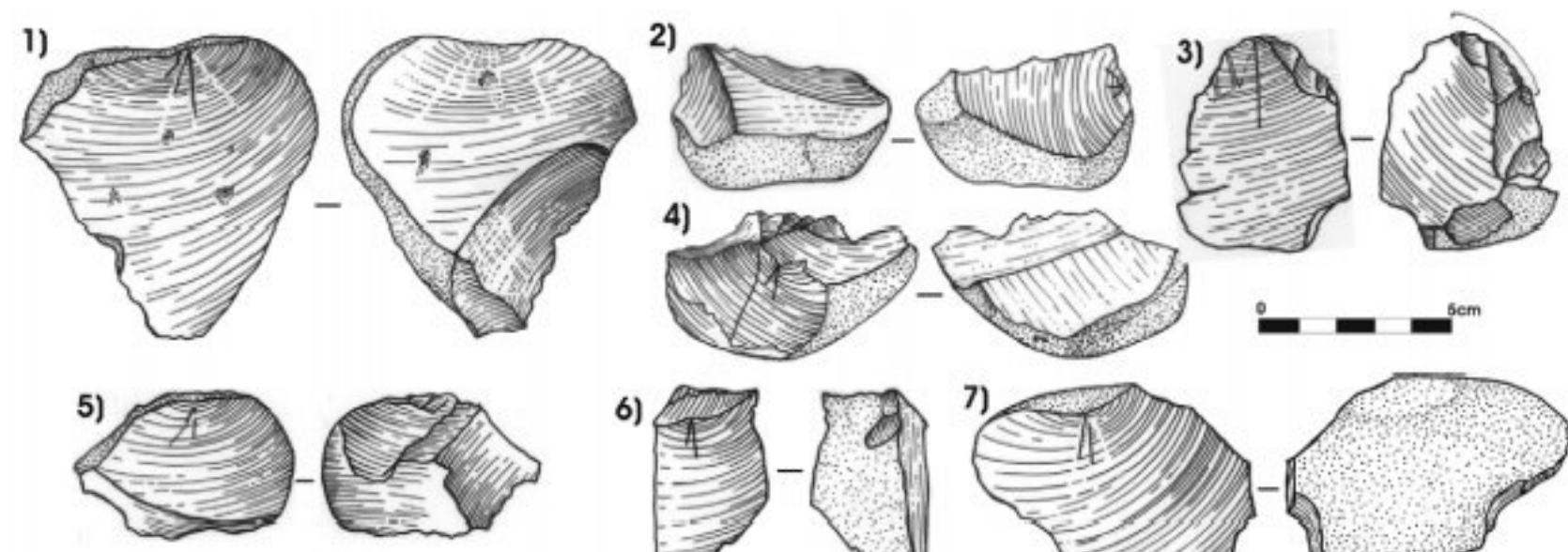

6)
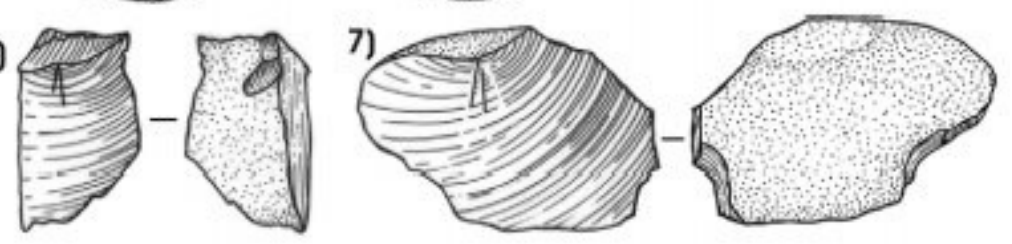

Figura 1: Dibujos de algunos de los materiales recuperados en OGS-7. Lascas completas $(1,3,5,6,7)$ y núcleos agotados (2,4). Fuente: Semaw et al., 2003.

\subsection{Dikika}

Las labores de prospección realizadas por parte del Dikika Research Project en los entornos de Andedo y Sidi Hakoma (Lower Awash, Etiopía), tuvieron como fruto los hallazgos en superficie de cuatro huesos con alteraciones tafonómicas interpretadas como marcas de corte. Los análisis realizados mediante microscopía electrónica de barrido (SEM), y derivados, concluyeron que las marcas de corte producidas en dos de los huesos, DIK-55-2 (un fragmento de costilla de un gran ungulado) y DIK-55-3 (un fragmento de fémur de un bóvido joven), fueron producidas en una fase previa a la fosilización (McPherron et al. 2010). La investigación determinó además que la morfología exhibida por las marcas no encajaba con las características propias de las alteraciones por trampling 2, sino que se correspondían con las generadas a partir de la utilización de herramientas líticas en actividades de descarnado y acceso a la médula ósea. Es necesario precisar el hecho de que los materiales de Dikika fueron recuperados en superficie, de forma descontextualizada, y que la datación de los mismos se realizó en base a un marcador calcáreo situado por encima de la pendiente donde fueron encontrados los huesos. La cronología estimada, pues, es de aproximadamente 3.39 Ma. (McPherron et al. 2010).

Las condiciones del hallazgo se encuentran rodeadas de polémica. En este sentido, las réplicas dirigidas a la investigación del Dikika Research Project se han concentrado fundamentalmente en discutir el contexto en el que los fósiles fueron recuperados, entendiendo que el hallazgo de un número tan escaso de restos en superficie no ofrece la solidez suficiente como para emitir juicios de semejante magnitud (Domínguez-Rodrigo, Pickering y Bunn, 2010, 2012). Los autores críticos niegan con rotundidad la verosimilitud de los resultados obtenidos tras el análisis tafonómico de DIK-55-2 y DIK-55-3, y han concluido, tras la elaboración de un programa experimental, que la morfología de las marcas que presentan la superficie de los huesos coincide con los patrones generados por trampling (Domínguez-Rodrigo et al., 2010, 2012).

De probarse como ciertas, las implicaciones que traen consigo los hallazgos de Dikika son muy importantes. Los $3.39 \mathrm{Ma}$. que plantean sus investigadores nos sitúan en una cronología prácticamente un millón de años más antigua que la datación ofrecida para Homo habilis. Durante muchas décadas la única especie documentada en la misma localización geográfica, en esa

2 En Tafonomía, el trampling o pisoteo se define como la alteración generada sobre la superficie de los huesos a partir de la abrasión que el sedimento genera sobre la cortical de los mismos. 
cronología concreta, fue Australopithecus afarensis, la cual no se encuentra adscrita a ningún contexto arqueológico y de la que no se infiere ningún tipo de comportamiento que implique el procesado de fauna. El reciente descubrimiento de Australopithecus deyiremeda (Haile-Selassie et al. 2015) en la región de Afar, no hace sino complicar el mapa paleoantropológico del Plioceno Medio.
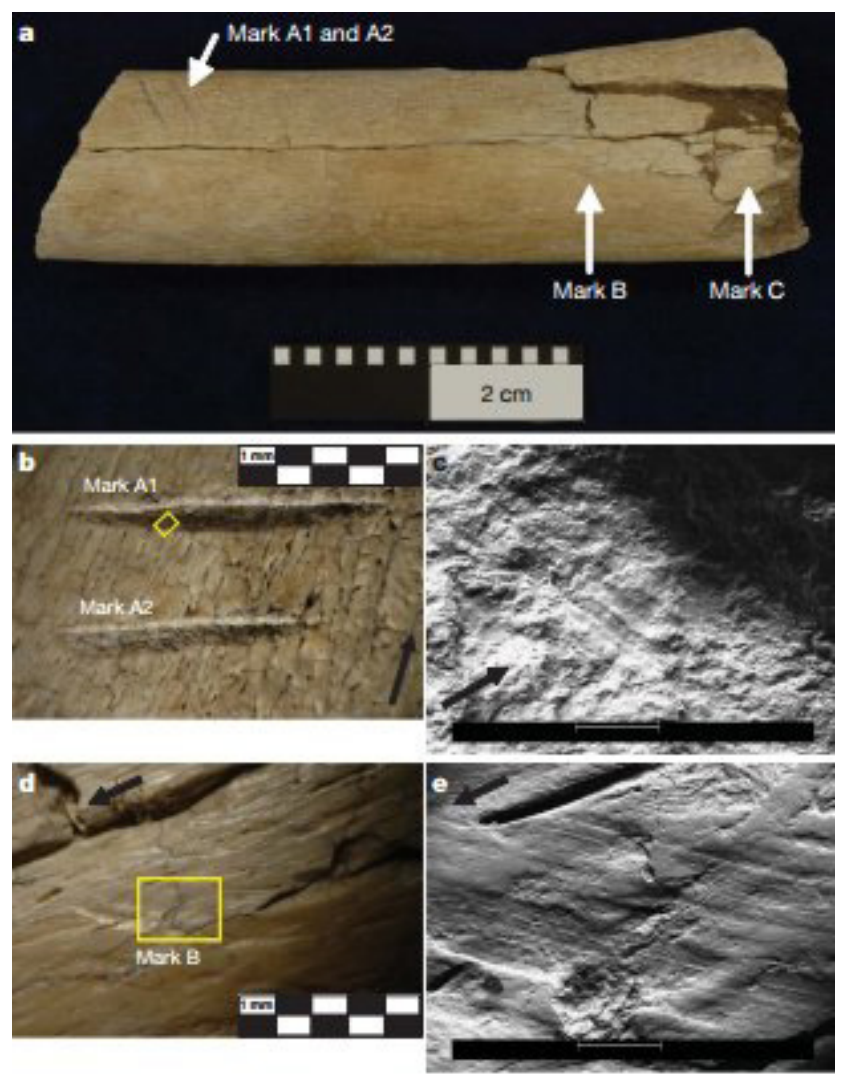

Figura 2: a: Estigmas identificados tafonomicamente como marcas de corte en la superficie de DIK-55- 2. b: Marcas A1 y A2 bajo microscopio óptico. c: Microestriaciones en las marcas A1 y A2, identificadas mediante el empleo de ESEM. d: Marca B bajo microscopio óptico. e: Microestriaciones en la marca B, identificadas mediante el empleo de ESEM. Fuente: McPherron et al., 2010.

\subsection{Lomekwi 3}

Las campañas de excavación realizadas entre 2011 y 2012 en el yacimiento de Lomekwi 3 (LOM 3, West Turkana, Kenya) han tenido como resultado el hallazgo de un número total de ciento cuarenta y nueve herramientas líticas recuperadas tanto en superficie como in situ (Harmand et al. 2015). El conjunto está compuesto por ochenta y tres núcleos, treinta y cinco lascas, siete yunques, siete percutores, tres cantos trabajados, dos cantos fracturados y doce piezas clasificadas como indeterminadas al carecer de elementos diagnósticos (Harmand et al. 2015). Las materias primas empleadas preferencialmente son basaltos, fonolitas y traquifonolitas. Los autores descartan el origen natural de las piezas, en base al análisis de los patrones de fracturación de las mismas. Por tanto, entienden el conjunto recuperado como el resultado de inequívocas actividades de débitage. A partir de esta idea, el West Turkana Archaeological Project ha desarrollado un programa experimental orientado a replicar la morfología de las herramientas de LOM 3 lo que, unido al análisis tecnológico, ha derivado en la identificación del empleo preferencial de la talla bipolar en el yacimiento (Harmand et al. 2015).

La datación de LOM 3 se ha obtenido a partir de muestras de paleomagnetismo procedentes del yacimiento, en correlación con dataciones radiométricas de tobas volcánicas pertenecientes a 
la formación geológica Nachukui. La cronología ofrecida para los materiales del sitio es de $3.3 \mathrm{Ma}$. (Harmand et al., 2015). Existe, sin embargo, un problema contextual intrínseco a la naturaleza del yacimiento, relacionado precisamente con la recuperación de una gran mayoría de las piezas en superficie (más de 100), así como la presencia de un depósito natural generado por la pendiente de la zona. Los propios autores de la investigación contemplan la redistribución de los materiales desde su posición primaria, esforzándose por relacionar los artefactos excavados con los encontrados en superficie (Harmand et al., 2015).

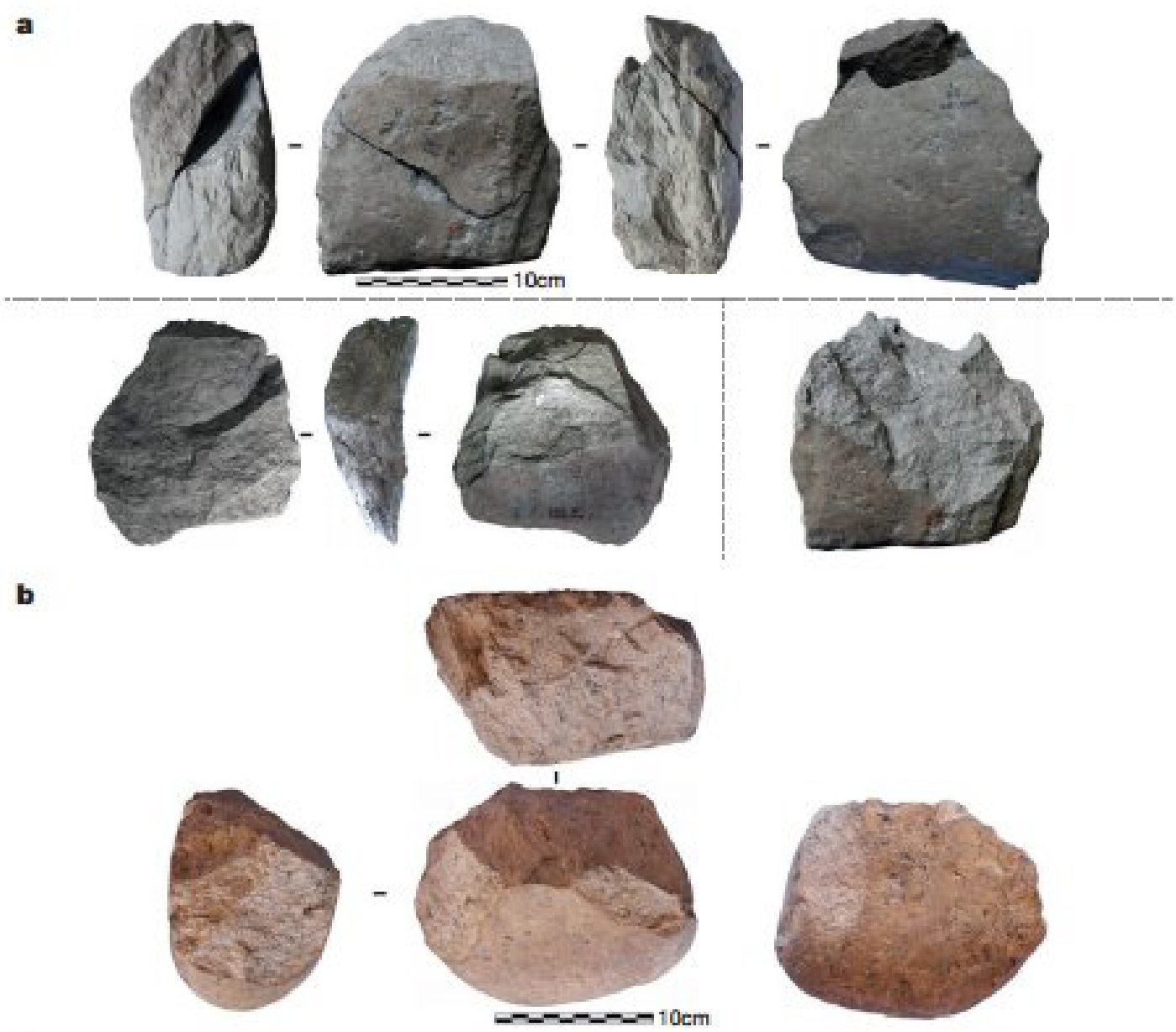

Figura 3: Muestra de algunos de los artefactos líticos recuperados en LOM3. a: Núcleo unifacial recuperado in situ, con remontaje de una lasca hallada en superficie. b: Núcleo unifacial recuperado in situ. Fuente: Harmand et al., 2015.

Estos resultados sitúan las actividades documentadas en Lomekwi en el contexto geográfico y cronológico de una única especie, Kenyanthropus platyops (Leakey et al., 2001), un polémico taxón que manifiesta una extraña mezcla de características anatómicas de los géneros Australopithecus y Homo (Cela Conde y Ayala, 2001).

\section{Discusión}

A partir de las conclusiones obtenidas por las investigaciones realizadas en el entorno de Gona, los planteamientos existentes sobre este conjunto de yacimientos resultan, a mi juicio, muy sólidos. El análisis de la industria de Modo 1 recuperada se complementa además con la presencia 
de restos de fauna asociados a la misma en OG-6 y OG-7. La proximidad geográfica y cronológica con Bouri y la comparación con sus materiales sirve también como apoyo. La aceptación por parte de la comunidad científica desde hace casi dos décadas convierte a Gona en la primera referencia cuando se aborda la cuestión de los orígenes de la tecnología.

La naturaleza del hallazgo de Dikika ha suscitado demasiada polémica como para que exista un consenso con respecto a la validez de las conclusiones que se derivan de sus resultados. Recientemente, un nuevo programa experimental ha tratado de probar la escasa semejanza de las alteraciones de los fósiles con las morfologías generadas a partir de trampling mencionadas anteriormente (Thompson et al., 2015). De cualquier modo, las condiciones del descubrimiento plantean graves problemas contextuales y de interpretación, que difícilmente permiten considerar la viabilidad de Dikika. Por otro lado, incluso asumiendo la naturaleza antrópica de las marcas de corte de DIK-55-2 y DIK-55-3 ¿realmente son reveladoras de una producción industrial por primitiva que esta sea? Los propios investigadores de Dikika son conscientes de que no ha de ser necesariamente así (McPherron et al., 2010). No es mi intención la de defender aquí la existencia de modelos pretecnológicos no sujetos a evidencias arqueológicas, pero ciertamente este es un debate que ha permanecido largas décadas sin cerrarse. Somos nosotros, quizás, en nuestras deficiencias formativas, quienes permanecemos incapaces de reconocer formas previas a la producción industrial que conocemos y, en consecuencia, valoramos.

El hallazgo del conjunto de Lomekwi 3 trae consigo unas implicaciones semejantes a las de Dikika, apoyadas en este caso por la actual recuperación de un conjunto de herramientas líticas. Retrasar la aparición de las industrias más antiguas hasta los más de 3.3 Ma. convertiría la producción tecnológica en una característica anterior a nuestro género, habiéndose datado en $2.8 \mathrm{Ma}$. el más arcaico ejemplar de Homo conocido. Por otro lado, gran parte de la propuesta del Lomekwiense como tecnocomplejo anterior al Olduvayense está fundamentada, según parece, en un argumento puramente cronológico. Los autores contemplan una menor complejidad técnica en los materiales de LOM 3 que en las características propias de la industria Olduvayense. Entienden, por tanto, que la naturaleza de la misma se relaciona con un modelo tecnológico anterior. Sin embargo, y juzgando únicamente a partir de los resultados publicados, apenas se aprecian diferencias con cualquier otro conjunto Olduvayense, con lo que la idea de establecer una distinción nominal y consecuentemente cultural podría resultar equívoca.

Cabría la posibilidad de pensar que lo que ha determinado la aparición de la producción tecnológica es el aumento de la capacidad craneal que manifiesta el género Homo con respecto a la línea de los australopitecinos. De acuerdo a esta idea, un mayor cerebro conllevaría una mayor encefalización, y de ella se derivaría la capacidad para generar una mayor complejidad técnica. $Y$ aunque recientemente se ha comprobado que, en efecto, el tamaño del cerebro importa en el caso de determinados mamíferos (Benson-Amram, Dantzer, Stricker, Swanson y Holekamp, 2016), al hablar de la capacidad para fabricar herramientas es necesaria la suma de más factores. No se trata, por tanto, únicamente de un aumento del tamaño cerebral, sino de la reorganización de sus áreas, especialmente de las cortezas premotora y motora, situadas en el lóbulo frontal, y que coordinan la gestión de movimientos y pensamientos complejos. En esa misma zona se localiza también el área de Broca, centro del habla que regula la producción y articulación de la misma. No en vano se ha relacionado extensamente la aparición del lenguaje con la capacidad de fabricar herramientas (Stout y Chaminade, 2009, 2012).

Moldes endocraneales realizados a fósiles del género Australopithecus muestran que la mencionada reorganización podría haberse originado en estadios previos a los $2 \mathrm{Ma}$. (Carlson et al. 2011; Falk et al., 2000) en una línea evolutiva que no posee una garantía de relación directa con la nuestra. La producción de herramientas se plantea, por lo tanto, como posible desde momentos anteriores y no ha de tratarse de una característica exclusiva del género Homo. Kada Gona se encuentra íntimamente ligada a los fósiles de Australopithecus garhi. Dikika se enmarca en las cronologías y en la geografía de Australopithecus afarensis y Australopithecus deyiremeda. Lomekwi ha sido testigo también del hallazgo del Kenyanthropus platyops, en las mismas cronologías. La 
génesis de Homo habilis se origina poco después, en tiempo geológico, de todos estos procesos. El horizonte paleoantropológico del momento plantea una enorme complejidad derivada de la gran cantidad de especies definidas en los últimos años (Plummer, 2004).

Por tanto, y entendiendo la diversidad de especies que han podido coexistir en un mismo territorio, junto a la posible capacidad para fabricar herramientas de las mismas: ¿funciona la tecnología como un criterio de humanidad válido y aplicable a nuestro género? Personalmente, la entiendo como si se tratase de una respuesta adaptativa al medio, una herramienta biológica con la que los grupos de homínidos se mostrarían competitivos. Y los recientes hallazgos plantean de forma cada vez más sólida que esa respuesta podría no ser exclusiva del género Homo. En términos de desarrollo cognitivo, la autoría de Australopithecus se plantea como una posibilidad real y a nivel arqueológico existen elementos suficientes como para generar una discusión, si bien los argumentos presentados en este trabajo no están exentos de dudas y detractores.

\section{Reflexiones finales}

Pese a que este tema pueda resultar controvertido y difícil de abordar, queda claro que existen dos tendencias en la investigación con respecto a los orígenes de la tecnología. La comunidad científica ha aceptado de forma prácticamente unánime la industria de Gona como la primera y más antigua de la que existe constancia. La autoría de la misma, aunque dudosa por la presencia de un creciente abanico de especies, resulta atribuible al género Homo. Los hallazgos y las publicaciones realizadas en los últimos años tienden a retrasar la aparición de la tecnología hasta los 3.3-3.4 Ma. convirtiéndola de ese modo en no exclusiva de nuestro género. Los yacimientos de Dikika y Lomekwi 3 plantean interesantes y novedosas propuestas, de enorme relevancia de confirmarse sus resultados. No hay que olvidar, no obstante, que ambos yacimientos están seriamente afectados por las condiciones en las que fueron realizados los descubrimientos. Sin embargo, los resultados generados a partir de la definición de nuevas especies y la proliferación de estudios cognitivos y primatológicos se ubican en una línea argumental clara: la producción de herramientas puede ser posible antes de nuestra aparición. Australopithecus, u otra especie dentro del complejo mapa paleoantropológico que compone el oriente de África en el límite cronológico del Plio-Pleistoceno, pudo tener esa capacidad, y ello ha de llevar necesariamente a una firme revisión de los criterios que nos definen como género.

\section{Bibliografía}

Asfaw, B., White, T., Lovejoy, O., Latimer, B., Simpson, S. y Suwa, G. (1999). Australopithecus garhi: a new species of early hominid from Ethiopia. Science, 284(5414), 629-635.

Benson-Amram, S., Dantzer, B., Stricker, G., Swanson, E. M. y Holekamp, K. E. (2016). Brain size predicts problem-solving ability in mammalian carnivores. Proceedings of the National Academy of Sciences, 11(9), 2532-2537.

Carlson, K. J., Stout, D., Jashashvili, T., De Ruiter, D. J., Tafforeau, P., Carlson, K. y Berger, L. R. (2011). The endocast of MH1, Australopithecus sediba. Science, 333(6048), 1402-1407.

Carvalho, S., Cunha, E., Sousa, C. y Matsuzawa, T. (2008). Chaînes opératoires and resourceexploitation strategies in chimpanzee (Pan troglodytes) nut cracking. Journal of Human Evolution, 55(1), 148-163.

Carvalho, S., Biro, D., McGrew, W. C. y Matsuzawa, T. (2009). Tool-composite reuse in wild chimpanzees (Pan troglodytes): archaeologically invisible steps in the technological evolution of early hominins?. Animal Cognition, 12(1), 103-114.

Castanedo, A. G. y Terreros de los, J. Y. S. (2009). Las herramientas de piedra más antiguas en África. Una visión general y algunas reflexiones. Sautuola: Revista del Instituto de Prehistoria y Arqueología. Sautuola, (15), 11-33. 
Cela Conde, C.J. y Ayala, F.J. (2001). Senderos de la evolución humana. Madrid: Alianza Editorial. Clark, G. (1977). World prehistory: in new perspective. Cambridge University Press.

Dart, R.A. (1957). The osteodontokeratic culture of Australopithecus prometheus (Vol. 10). Transvaal Museum.

Dart, R.A. y Wolberg, D.L. (1971) On the Osteodontokeratic Culture of the Australopithecinae. Current Anthropology 12(2), 233-236.

Delagnes, A. y Roche, H. (2005). Late Pliocene hominid knapping skills: the case of Lokalalei 2C, West Turkana, Kenya. Journal of Human Evolution, 48(5), 435-472.

Díez Martin, F. (2003). La aplicación de los "modos tecnológicos" en el análisis de las industrias paleolíticas. Reflexiones desde la perspectiva europea. SPAL, 12, 35-51.

Domínguez-Rodrigo, M., Pickering, T. R. y Bunn, H. T. (2010). Configurational approach to identifying the earliest hominin butchers. Proceedings of the National Academy of Sciences, 107(49), 20929-20934.

Domínguez-Rodrigo, M., Pickering, T. R. y Bunn, H. T. (2012). Experimental study of cut marks made with rocks unmodified by human flaking and its bearing on claims of 4-million-year-old butchery evidence from Dikika, Ethiopia. Journal of Archaeological Science. 39(2), 205-214.

Falk, D., Redmond, J. C., Guyer, J., Conroy, C., Recheis, W., Weber, G. W. y Seidler, H. (2000). Early hominid brain evolution: a new look at old endocasts. Journal of Human Evolution, 38(5), 695-717.

Haile-Selassie, Y., Gibert, L., Melillo, S. M., Ryan, T. M., Alene, M., Deino, A., ... y Saylor, B. Z. (2015). New species from Ethiopia further expands Middle Pliocene hominin diversity. Nature, 521(7553), 483-488.

Haslam, M., Hernandez-Aguilar, A., Ling, V., Carvalho, S., de La Torre, I., DeStefano, A., ... y Matsuzawa, T. (2009). Primate archaeology. Nature, 460(7253), 339-344.

Harmand, S., Lewis, J. E., Feibel, C. S., Lepre, C. J., Prat, S., Lenoble, A., ... y Taylor, N. (2015). 3.3-million-year-old stone tools from Lomekwi 3, West Turkana, Kenya. Nature, 521(7552), 310-315.

Heinzelin de, J., Clark, J. D., White, T., Hart, W., Renne, P., WoldeGabriel, G., ... y Vrba, E. (1999). Environment and behavior of 2.5-million-year-old Bouri hominids. Science, 284(5414), 625629.

Inoue-Nakamura, N. y Matsuzawa, T. (1997). Development of stone tool use by wild chimpanzees (Pan troglodytes). Journal of comparative psychology, 111(2), 159.

Leakey, L. S. B., Tobias, P. V. y Napier, J. R. (1964) A new species of the genus Homo from the Olduvai Gorge. Nature. 202(4927), 7-9.

Leakey, M. G., Spoor, F., Brown, F. H., Gathogo, P. N., Kiarie, C., Leakey, L. N. y McDougall, I. (2001). New hominin genus from eastern Africa shows diverse middle Pliocene lineages. Nature, 410(6827), 433-440.

McPherron, S. P., Alemseged, Z., Marean, C. W., Wynn, J. G., Reed, D., Geraads, D., ... y Béarat, H. A. (2010). Evidence for stone-tool-assisted consumption of animal tissues before 3.39 million years ago at Dikika, Ethiopia. Nature, 466(7308), 857-860.

Mercader, J., Panger, M. y Boesch, C. (2002). Excavation of a chimpanzee stone tool site in the African rainforest. Science. 296(5572), 1452-1455.

McGrew, W.C. (1992). Chimpanzee material culture: implications for human evolution. Cambridge: Cambridge University Press.

Panger, M. A., Brooks, A. S., Richmond, B. G. y Wood, B. (2002). Older than the Oldowan? Rethinking the emergence of hominin tool use. Evolutionary Anthropology: Issues, News, and Reviews, 11(6), 235-245.

Plummer, T. (2004). Flaked stones and old bones: biological and cultural evolution at the dawn of technology. American Journal of Physical Anthropology. 125(S39), 118-164.

Plummer, T., Bishop, L. C., Ditchfield, P. y Hicks, J. (1999). Research on Late Pliocene Oldowan sites at Kanjera South, Kenya. Journal of Human Evolution, 36(2), 151-170. 
Semaw, S. (2000). The world's oldest stone artefacts from Gona, Ethiopia: their implications for understanding stone technology and patterns of human evolution between 2.6-1.5 million years ago. Journal of Archaeological Science, 27(12), 1197-1214.

Semaw, S., Renne, P., Harris, J. W., Feibel, C. S., Bernor, R. L., Fesseha, N. y Mowbray, K. (1997). 2.5-million-year-old stone tools from Gona, Ethiopia. Nature, 385(23), 333-336

Semaw, S., Rogers, M. J., Quade, J., Renne, P. R., Butler, R. F., Dominguez-Rodrigo, M., ... y Simpson, S. W. (2003). 2.6-Million-year-old stone tools and associated bones from OGS-6 and OGS-7, Gona, Afar, Ethiopia. Journal of Human Evolution, 45(2), 169-177.

Schwartz, J. H. y Tattersall, I. (2015). Defining the genus Homo. Science, 349(6251), 931-932.

Stout, D., Quade, J., Semaw, S., Rogers, M. J. y Levin, N. E. (2005). Raw material selectivity of the earliest stone toolmakers at Gona, Afar, Ethiopia. Journal of Human Evolution, 48(4), 365-380.

Stout, D. y Chaminade, T. (2009). Making tools and making sense: complex, intentional behaviour in human evolution. Cambridge Archaeological Journal. 19(01), 85-96.

Stout, D. y Chaminade, T. (2012). Stone tools, language and the brain in human evolution. Philosophical Transactions of the Royal Society of London B: Biological Sciences. 367(1585), 75-87.

Thompson, J. C., McPherron, S. P., Bobe, R., Reed, D., Barr, W. A., Wynn, J. G., ... y Alemseged, Z. (2015). Taphonomy of fossils from the hominin-bearing deposits at Dikika, Ethiopia. Journal of human evolution, 86, 112-135.

Torre de la, I. (2011). The origins of stone tool technology inAfrica: a historical perspective. Philosophical Transactions of the Royal Society of London B: Biological Sciences, 366(1567), 1028-1037.

Villmoare, B., Kimbel, W. H., Seyoum, C., Campisano, C. J., DiMaggio, E. N., Rowan, J., ... y Reed, K. E. (2015). Early Homo at 2.8 Ma from Ledi-Geraru, Afar, Ethiopia. Science, 347(6228), $1352-1355$.

White, L. A. (1959). The concept of culture. American anthropologist. 61(2), 227-251. 


\section{Panta Rei}

PANTA REI es una revista digital de investigación orientada a la Historia y otras ciencias afines. Su principal objetivo es la transmisión del conocimiento científico, dando una oportunidad también a los jóvenes investigadores que quieren abrirse camino en el estudio de las ciencias humanas y sociales. Se compone de estudios originales relacionados con la disciplina histórica así como su didáctica y difusión. Las diferentes secciones que componen la revista son: artículos de investigación, entrevistas a profesionales, recensiones de monografías de actualidad y crónicas de congresos o eventos científicos relevantes.

Todos los artículos publicados son objeto de un proceso de revisión a cargo de un mínimo de dos evaluadores, que se consideran expertos en el ámbito temático del artículo propuesto. Nuestro deseo es poder ofrecer unos contenidos rigurosos, de calidad y de interés.

EI CEPOAT (Centro de Estudios del Próximo Oriente y la Antigüedad Tardía de la Universidad de Murcia) es la institución encargada de la coordinación y gestión de la revista, desde donde anualmente se lanzará la convocatoria para aquellos que estén interesados en publicar sus trabajos, siempre relacionados con la Historia, Arqueología, Historia del Arte, Didáctica de las Ciencias Sociales, etc.

PANTA REI is a digital journal focused on History and other sciences related to it. Its main objective is the transmission of scientific knowledge by giving also an opportunity to young researchers who want to make their way in the study of human and social sciences. It is composed by original studies related to History, as well as its didactics and promotion. The different sections of this journal are: research articles, interviews to professionals, recensions on monographs about current issues and reports about congresses or relevant scientific events.

All the articles published are subject to a revision process carried out by a minimum of two reviewers who are considered to be experts in the field of the article proposed. Our wish is to offer rigorous contents with quality and being of interest to the reader.

CEPOAT (Centre of Studies of the Middle East and Late Antiquity of the University of Murcia) is the institution in charge of the coordination and management of this journal. This is the centre from where the call for papers will be launched for all the people interested in publishing their papers, always related to History, Archeology, Art History, Didactics of the Social Sciences, etc. 


\section{Normas de Publicación}

El autor se compromete a enviar trabajos originales, que no se encuentren publicados en otras revistas ni en otros idiomas. Así mismo, el mismo artículo no podrá ser presentado en otras revistas mientras dure el proceso de evaluación.

\section{Envío y presentación de originales}

Los artículos se enviarán exclusivamente a través del correo electrónico a la dirección pantarei@um.es. Los textos serán enviados en formato DOC y las imágenes en formato JPEG o TIFF, y con un tamaño mínimo de 2000 px. Éstas no aparecerán incorporadas en el texto, sino enviadas en archivo aparte y correctamente numeradas según su posición en el texto. Junto al trabajo, se rellenará y enviará un documento aparte en el que se especifiquen los datos del autor siguiendo el modelo disponible en la página Web de la revista.

Para la redacción de los trabajos se tendrá en cuenta el Manual de la American Psychological Association, en su sexta edición. La extensión máxima de los trabajos será de 30 páginas. La tipografía será Arial 11, con interlineado sencillo y sin espacio alguno entre párrafos. El texto deberá ir justificado a ambos márgenes y sin sangría en los primeros párrafos. Los márgenes serán de $2,50 \mathrm{~cm}$. En los casos en los que fuera necesario incorporar notas, éstas irán a pie de página, enumeradas consecutivamente, con tipografía Arial 10, interlineado sencillo y justificadas a ambos márgenes.

Una información más detallada se encuentra disponible en la página http://www.um.es/cepoat/ pantarei.

\section{Proceso de valoración y evaluación}

Una vez recibidos los trabajos, la Revista realizará una primera valoración. Si el trabajo enviado se ajusta a las normas de presentación propuestas, la temática es coincidente con la línea editorial de la revista y posee la calidad científica necesaria, será remitido al consejo asesor para una primera evaluación. Si no es así en este primer paso se puede rechazar directamente los documentos que incumplan claramente la línea editorial.

Será el Consejo Asesor quien indique a la revista la originalidad, relevancia, estructura, redacción, aparato bibliográfico, etc. del trabajo enviado y, para ello, se designará a dos revisores expertos externos que evaluarán cada uno de los trabajos, que pueden formar parte (o no) de este Consejo Asesor. La selección de los revisores se ajustará a la temática y características metodológicas del trabajo. El nombre y filiación de los autores serán eliminados del trabajo para su revisión, así como los revisores actuarán de manera anónima y confidencial.

Los revisores deberán rellenar un informe de evaluación que centrará su atención en aspectos tales como características formales, originalidad y novedad de los trabajos, relevancia de las propuestas y los resultados, calidad metodológica y validez científica.

Una vez terminado el proceso se decidirá la aceptación o no de los mismos y su publicación en el número que sea pertinente, así como las modificaciones susceptibles de ser realizadas para su final publicación. Dicha notificación se enviará únicamente por correo electrónico, en un plazo máximo de seis meses. 


\section{Publishing rules}

The author is committed to submit original papers not having been published in other reviews or in other languages. In this way, it is not allowed for the same paper to be presented in other reviews during the evaluation process.

\section{Submission and presentation of originals}

The articles will be exclusively submitted by email to pantarei@um.es. The texts will be submitted in DOC format and the images in JPEG or TIFF format, and with a minimum size of 2000 px. Images will not be integrated in the text but sent in another file and properly numbered according to their position in the text. Attached to the paper, a document will be filled out and sent where the author's data will be specified following the model available on the website.

The sixth edition of the Manual of the American Psychological Association will be taken into account for the writing of the papers. The length of the papers must not exceed 30 pages. Typography will be Arial 11 , with simple line spacing and no space between paragraphs. The text must be justified on both margins without indentation in the first paragraphs. Margins size will be $2.50 \mathrm{~cm}$. Where it could be necessary the incorporation of notes, they will be at the bottom of the page, consecutively numbered with typography Arial 10, simple line spacing and justified on both margins.

More detailed information is available on the website: http://www.um.es/cepoat/pantarei.

\section{Examination and assessment process}

The Journal will submit the papers to a first examination once received. If the paper follows the presentation guidelines, the subject agrees with the editorial line of this journal, and possess the scientific quality required, it will be sent to the advisory council for a first assessment. If not, the documents which clearly fail to complete the editorial line may be rejected straightaway in this first step.

The Advisory Council will indicate the originality, relevance, structure, writing, bibliography, etc. of the text to the journal; for this purpose, two outside experts will be designated to review the papers; these experts can be (or not) part of this Advisory Council. The selection of the experts will adjust to the subject and methodological characteristics of the paper. Name and affiliation of the author will be eliminated from the text for its review, in this way experts will act anonymously and confidentially.

The experts will fill out an assessment report which will focus on aspects such as formal characteristics, originality and novelty of the papers, relevance and results of the proposal, methodological quality and scientific validity.

Once the process is finished, the acceptance or not of the papers and its publication in the corresponding edition will be decided, as well as the modifications that may be done for its final publication. This notification will be sent by email within 6 months maximum. 


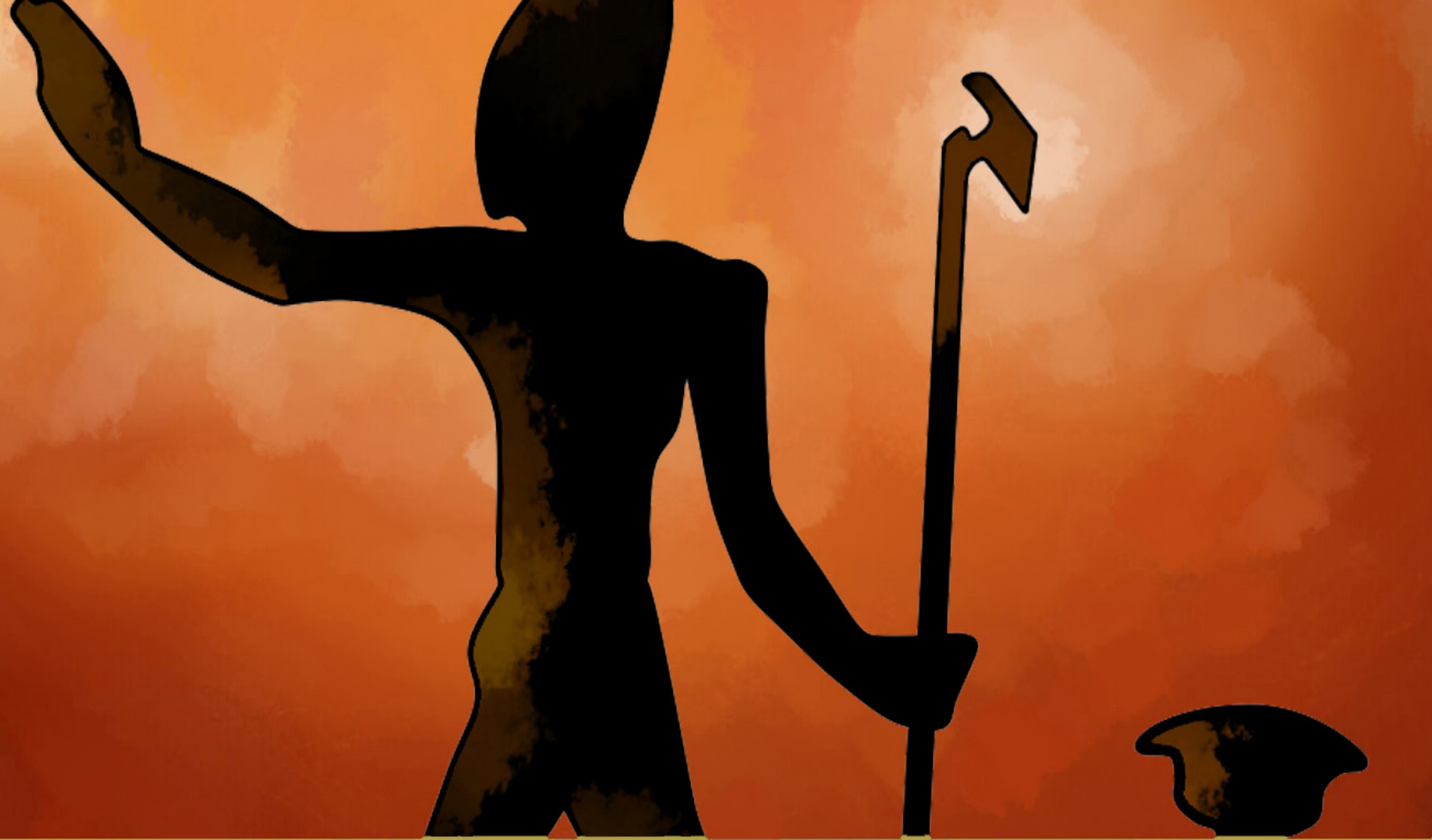

cepo t

UNIVERSIDAD DE MURCIA

centro de estudios del

próximo oriente y la

antigüedad tardía 